\title{
Impact of Thermodiffusion on the Initial Vertical Distribution of Species in Hydrocarbon Reservoirs
}

\author{
Guillaume Galliero $^{1} \cdot$ Henri Bataller $^{1} \cdot$ Fabrizio Croccolo $^{1} \cdot$ Romain $_{\text {Vermorel }}{ }^{1}$. \\ Pierre-Arnaud Artola ${ }^{2}$. Bernard Rousseau ${ }^{2}$. Velisa Vesovic ${ }^{3}$. Mounir Bou-Ali ${ }^{4}$. \\ José M. Ortiz de Zárate ${ }^{5}$. Shenghua $\mathrm{Xu}^{6} \cdot \mathrm{Ke}^{\mathrm{Zhang}}{ }^{7} \cdot$ François Montel $^{8}$
}

Received: 26 May 2015 / Accepted: 19 October 2015 / Published online: 4 November 2015

(C) Springer Science+Business Media Dordrecht 2015

\begin{abstract}
In this work we propose a methodology, based on molecular dynamics simulations, to quantify the influence of segregation and thermodiffusion on the initial state distribution of the fluid species in hydrocarbon reservoirs. This convection-free approach has been applied to a synthetic oil composed of three normal alkanes and to a real acid gas. It has been found that the thermodiffusion effect induced by the geothermal gradient is similar (but opposite in sign) to that due to segregation for both mixtures. In addition, because of the combined effect of thermal expansion
\end{abstract}

Guillaume Galliero

guillaume.galliero@univ-pau.fr

1 Laboratoire des Fluides Complexes et leurs Réservoirs, UMR-5150 CNRS-TOTAL, Université de Pau et des Pays de l'Adour, Pau, France

2 Laboratoire de Chimie-Physique, UMR 8000 CNRS, Université Paris-Sud, Orsay, France

3 Department of Earth Science and Engineering, Imperial College London, London, UK

4 MGEP Mondragon Goi Eskola Politeknikoa, Mechanical and Industrial Manufacturing Department, Mondragon, Spain

5 Departamento de Física Aplicada I, Universidad Complutense, Madrid, Spain

6 Key Laboratory of Microgravity, Institute of Mechanics, Chinese Academy of Science, Beijing, China

7 State Key Laboratory of Enhanced Oil Recovery, CNPC, Beijing, China

8 TOTAL E.P., Pau, France and thermodiffusion, it has been observed that the density gradient can be reversed, in the presence of a geothermal gradient. These numerical results emphasize the need of improving our quantification of thermodiffusion in multicomponent mixtures. The SCCO-SJ10 experiments will be a crucial step towards this goal.

Keywords Thermodiffusion - Segregation ·

Multicomponent mixtures · Oil and gas · Molecular dynamics

\section{Introduction}

Compositional grading has a strong impact on the estimation of the initial (before production) hydrocarbon in place and is important for selecting the best reservoir development scenario. In a closed, convection free reservoir, the compositional variations are mainly determined by gravitational segregation (Høier and Whitson 2001). However, other phenomena may affect the compositional grading in real reservoirs (Holt et al. 1983; Whitson and Belery 1994; Montel et al. 2007). Among them is thermodiffusion, or Soret effect, induced by the geothermal gradient (Ghorayeb et al. 2003; Montel et al. 2007), which is a phenomenon that couples heat and mass fluxes (de Groot and Mazur 1984). The contribution of thermodiffusion is difficult to quantify, mainly due to a lack of experimental data as well as accurate modelling for multi-component mixtures. Although noticeable progresses have been made during the last twenty years, see Assael et al. (2014) and references therein, especially on ternary mixtures both theoretically (Firoozabadi et al. 2000; Kempers 2001; Galliero et al. 2003) and experimentally 
(Leahy-Dios et al. 2005; Bou-Ali et al. 2015) more work is necessary. Micro-gravity experiments are one possible way to provide further data on thermodiffusion in multicomponent mixtures (Georis et al. 1998; Van Vaerenbergh et al. 2009; Touzet et al. 2011; Khlybov et al. 2015). They are particularly interesting because they provide, by default, experimental conditions free of convection effect.

The need for further advancement in the field motivated the development of a project named Soret Coefficient measurements of Crude Oil in SJ10 (SCCO-SJ10). This project aims at measuring thermodiffusion in multicomponent fluid mixtures of petroleum interest using a microgravity set-up. It follows a previous SCCO experiment that has flown on Russian Foton M3 missions (Van Vaerenbergh et al. 2009; Touzet et al. 2011). This previous experiment was dedicated to the measurement of thermodiffusion in binary, ternary and quaternary hydrocarbon "academic" mixtures. It has yield interesting results in liquid mixtures showing, among others, the limitations of some models to quantify thermodiffusion in more than binary mixtures (Van Vaerenbergh et al. 2009). However, for the mixtures composed of gases and liquids, difficulties were encountered during the gas chromatography analysis leading to unexpectedly high values of the measured thermodiffusion separation (Touzet et al. 2011).

The SCCO-SJ10 project is made possible as a result of a partnership between European Space Agency and China's National Space Science Center (Hu et al. 2014). The microgravity experiment is scheduled to fly on China's SJ-10 Shi Jian spacecraft during April 2016 (Hu et al. 2014). In addition to the two space agencies, this project involves academics from France (Université de Pau et des Pays de l'Adour, Université de Paris-Sud), Spain (Mondragon Unibertsitatea, Universidad Complutense), United Kingdom (Imperial College London), China (Chinese Academy of Sciences) and industrials from France (Total) and China (RIPED). The microgravity set-up will consist of six cells designed by Sanchez Technology (Georis et al. 1998). Each cell will contain a different mixture ( 3 of "academic" interest and 3 of "industrial" interest) under high pressures, up to $40 \mathrm{MPa}$, at an average temperature of $50{ }^{\circ} \mathrm{C}$, chosen to mimic reservoir conditions. The filling, as well as the post-flight analysis, of the cells will be performed by RIPED.

To complement this micro-gravity experiment, various on-ground activities will be carried on. They will consist of:

- ground experiments (Croccolo et al. 2012; Urteaga et al. 2012; Giraudet et al. 2014; Larrañaga et al. 2015) on thermodiffusion in ternary mixtures that will take advantage of an extension of the theory of nonequilibrium fluctuations in ternary mixtures (Ortiz de Zárate et al. 2014),
- Molecular Dynamics (MD) simulations of thermodiffusion in multicomponent mixtures in bulk (Galliero et al. 2003; Galliero et al. 2009; Artola and Rousseau 2013) and confined situations (Galliero et al. 2006; Hannaoui et al. 2013),

- developing models to take into account thermodiffusion when describing the initial state of a reservoir (Montel et al. 2007; Touzet et al. 2011).

Within this framework, the purpose of the current article is to describe a numerical approach emphasizing the influence of thermodiffusion on the vertical distribution of species at the initial state of hydrocarbon reservoirs. The approach relies on MD simulations and on classical thermodynamic modelling based on an Equation of State (EoS) as described in Galliero and Montel (2008). The main focus of this article is on the influence of gravitational and geothermal fields in a convection free configuration. We have neglected the influence of the porous media on segregation and thermodiffusion which is a reasonable assumption at the steady state providing the permeability is not too low (Shapiro and Stenby 2000; Platten and Costeseque 2004; Montel et al. 2007; Hannaoui et al. 2013). To apply this approach, two mixtures have been selected, a synthetic one composed of methane, $n$-butane and $n$-dodecane $\left(\mathrm{C}_{1}-\mathrm{nC}_{4}\right.$ $\mathrm{nC}_{12}$ ), and one representing a realistic acid gas, rich in carbon dioxide and lean in hydrogen sulphide.

\section{Theory and Methods}

\section{Thermo-Gravitation}

A gravitational field applied to a convection free fluid mixture leads to a partial separation of the species along the vertical axis (de Groot and Mazur 1984). This phenomenon is usually named gravitational segregation and its dynamics is controlled by mass diffusion (Galliero and Montel 2008). At equilibrium, the isothermal vertical distribution (assuming a one dimensional reservoir) of the species $i$ can be deduced from (Sage and Lacey 1939):

$$
\left(\frac{d \mu_{i}}{d z}\right)_{T}=M_{i} g
$$

where $\mu_{i}$ and $M_{i}$ are the chemical potential and the molecular weight of species $i$, respectively, $z$ is the vertical position, $T$ the temperature and $g$ the gravitational acceleration. This equation, given a composition at a reference point $z^{0}$, can be used to obtain the variation of composition at the stationary state as a function of depth. However, except for ideal mixtures, such as isotope ones (Galliero and Montel 2008), Eq. 1 cannot be solved analytically and a thermodynamic 
modeling combined with a numerical procedure has to be employed (Montel and Gouel 1985; Halldórsson and Stenby 2000).

When a vertical geothermal gradient is added (the fluid column is heated from below), at the stationary state the distribution of the species $i$ can be obtained from an extension of the Gibbs equation (Galliero and Montel 2008):

$\left(\frac{d \mu_{i}}{d z}\right)_{T}=M_{i} g-\frac{\alpha_{T_{i}}}{T} \frac{d T}{d z}$

where $\alpha_{T_{i}}$ is the "generalized" thermal diffusion factor of component $i$ (Kempers 2001). Such an equation also cannot be solved analytically, except when dealing with ideal mixtures.

\section{Molecular Dynamics Simulations}

The classical molecular dynamics method consists in numerically estimating the positions, velocities and acceleration of a number of selected molecules evolving in space and time. The motions of the molecules are described through a numerical integration of the classical Newton's law equations while the interactions between molecules are described by effective potentials (Allen and Tildesley 1987). This method can be used to study both equilibrium and transport properties of model fluids, mimicking real ones.

\section{Fluid Models}

To model the species present in the selected alkane mixture $\left(\mathrm{C}_{1}, \mathrm{nC}_{4}, \mathrm{nC}_{12}\right)$, the simple Lennard-Jones (LJ) chain model has been employed (Galliero 2014). The molecules are represented by $N$ freely jointed tangent spheres and nonbonded interaction between spheres $i$ and $j$ is described by the usual Lennard-Jones 12-6 potential:

$U_{i j}^{L J}=4 \varepsilon_{i j}\left[\left(\frac{\sigma_{i j}}{r_{i j}}\right)^{12}-\left(\frac{\sigma_{i j}}{r_{i j}}\right)^{6}\right]$

where $\sigma_{i j}$ is the distance at which the potential is equal to zero (the "sphere diameter"), $\varepsilon_{i j}$ the potential depth and $r_{i j}$ the distance between spheres $i$ and $j$. Concerning bonded interaction, two adjacent spheres are connected by a constrained bond of a length equal to $\sigma$.
To describe the polar compounds, i.e. $\mathrm{H}_{2} \mathrm{~S}$ and $\mathrm{CO}_{2}$, a single $\mathrm{LJ}$ sphere combined with a simple isotropic multipolar potential (IMP) has been employed (Galliero et al. 2007a):

$U_{i j}^{I M P}=U_{i j}^{L J}-\frac{1}{k_{B} T}\left[\frac{p_{i}^{2} p_{j}^{2}}{3 r_{i j}^{6}}+\frac{p_{i}^{2} Q_{j}^{2}+p_{j}^{2} Q_{i}^{2}}{2 r_{i j}^{8}}+\frac{7 Q_{i}^{2} Q_{j}^{2}}{5 r_{i j}^{10}}\right]$

where $p_{i}$ is the dipole moment of molecule $i$ and $Q_{i}$ the quadrupole moment of molecule $i$.

A cutoff radius equal to $3.5 \sigma_{i j}$ has been used during simulations and long range corrections have been included (Allen and Tildesley 1987). Cross parameters between molecules of different species have been deduced using the classical Lorentz-Berthelot combining rules.

The molecular parameters, provided in Table 1, have been adjusted using the pure component properties, including equilibrium vapour-liquid phase envelope and viscosities (Galliero et al. 2007a; Touzet et al. 2011).

\section{Thermo-Gravitation MD Algorithm}

To simulate a one dimensional gravitational segregation process using MD, the simulation box is divided into $N_{S}$ slabs along the vertical direction. Then, a vertical "gravitational" force equal to $-M_{i} g_{M D}$ is applied on each molecule belonging to slabs 1 to $N_{S} / 2$ (upper half of the simulation box), whereas a vertical force equal to $+M_{i} g_{M D}$ is applied to each molecule belonging to slabs $N_{S} / 2+1$ to $N_{S}$ (lower half of the simulation box), see Fig. 1. By doing so, a bi-periodical pressure gradient is induced in the simulation box and the segregation process can take place.

The height, $h_{M D}$, see Fig. 1, used in the molecular simulations is typically of the order of $10 \mathrm{~nm}$. So, to mimic the real size of a petroleum reservoir the amplitude, $g_{M D}$, of the "gravitational" force is chosen so that the product $g_{M D} h_{M D}$ is equal to $g h$ of a target real reservoir (where $g$ is the gravitational acceleration and $h$ is of the order of hundreds of meters), i.e. in the simulations $g_{M D} \approx 10^{11} \mathrm{~m} . \mathrm{s}^{-2}$ depending on the system studied. This approach is consistent as long as the response remains linear and if there are no fluid-fluid interfaces appearing in the system.

Table 1 Molecular parameters employed in this work

\begin{tabular}{llllll}
\hline Species & $N$ & $\varepsilon\left(\mathrm{J}^{\mathrm{mol}}{ }^{-1}\right)$ & $\sigma(\AA)$ & $p(\mathrm{D})$ & $Q(\mathrm{D} . \AA)$ \\
\hline $\mathrm{C}_{1}$ & 1 & 1246.5 & 3.7327 & 0 & 0 \\
$\mathrm{nC}_{4}$ & 2 & 2075 & 3.979 & 0 & 0 \\
$\mathrm{nC}_{12}$ & 5 & 2490 & 4.042 & 0 & 0 \\
$\mathrm{H}_{2} \mathrm{~S}$ & 1 & 2320 & 3.688 & 0.9 & 0 \\
$\mathrm{CO}_{2}$ & 1 & 1619 & 3.758 & 0 & 4.0982 \\
\hline
\end{tabular}




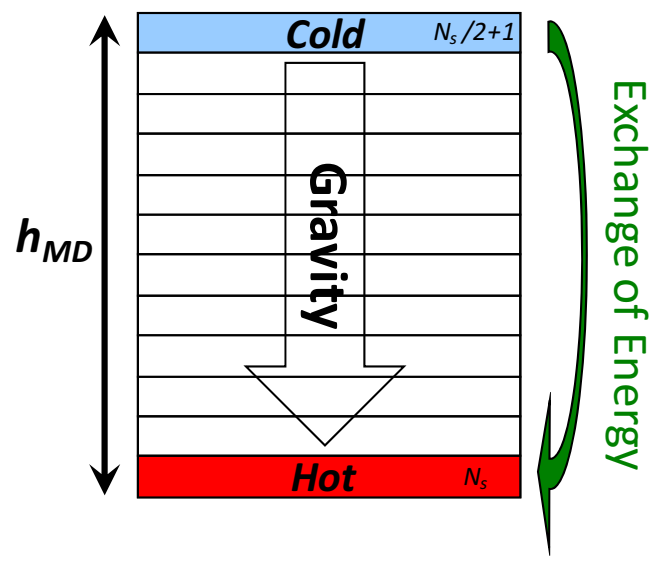

Fig. 1 A sketch of the scheme employed to study thermogravitation using MD simulations (only the lower half of the simulation box is shown)

To impose a geothermal gradient (taken here to be around $0.025-0.03 \mathrm{~K} . \mathrm{m}^{-1}$ as typically found in real petroleum reservoirs), a bi-periodical thermal gradient is applied to the simulation box by using the non-equilibrium algorithm proposed by Müller-Plathe (1997). This scheme consists in exchanging kinetic energy between the edge layers and the central layers of the simulation box as shown in Fig. 1. As for the gravity field the temperature gradient applied has been chosen to represent the one applied on the simulated reservoir column, resulting in huge thermal gradients of the order of $10^{9} \mathrm{~K} \cdot \mathrm{m}^{-1}$. However, in line with previous studies (Perronace et al. 2002; Galliero and Montel 2008), the response of the system was found to be always linear. In addition, in a previous work (Galliero and Montel 2008), a similar approach has been validated on "isotopic" mixtures for which an analytical solution exists.

It is important to note that because the fluid column is heated from below, which corresponds to a RayleighBénard configuration, the system may be unstable and convection may occur at the reservoir scale (Montel et al. 2007). However, during MD simulations, the Rayleigh number remains always below its critical threshold because of the small size $(\mathrm{nm})$ of the simulation domain (Galliero and Montel 2008). Thus, the fluid column simulated by this approach is always stable (convection free) and only diffusive processes take place.

\section{Simulations Details}

Using a homemade MD code, systems composed of 2000 to 4000 molecules have been simulated. The velocity Verlet algorithm has been applied to integrate the equation of motion (Allen and Tildesley 1987). A reduced timestep, $\delta t^{*}$, equal to 0.003 has been used. To maintain the desired average temperature during simulations, a Berendsen thermostat with a large time constant equal to $1000 \delta t^{*}$ has been utilized (Berendsen et al. 1984). Classical periodic boundary conditions combined with a Verlet neighbors list have been applied (Allen and Tildesley 1987). Once the stationary state is reached, data have been collected and averaged during simulations lasting fifty millions time steps. Slabs 1, $2, N_{s} / 2-1, N_{s} / 2, N_{s} / 2+1, N_{s} / 2+2, N_{s}-1$ and $N_{s}$ have been discarded when performing the analysis of the MD concentrations, temperature and density profiles. Error bars, computed using the sub-block method (Allen and Tildesley 1987), are of the order of \pm 0.01 on mole fraction, $\pm 0.1 \mathrm{~K}$ on temperature and $\pm 0.2 \mathrm{~kg} / \mathrm{m}^{3}$ on density. They have been omitted in the figures, for sake of clarity.

\section{Results and Discussion}

\section{Synthetic Mixture Composed of Normal Alkanes}

As a first example we have studied the behavior of a $\mathrm{C}_{1}$ $\mathrm{nC}_{4}-\mathrm{nC}_{12}$ synthetic mixture in which the mole fractions are respectively 0.2, 0.4 and 0.4 (Van Vaerenbergh et al. 2009). Reference conditions at the mid column depth are a temperature equal to $333.15 \mathrm{~K}$ and a pressure equal to 35 MPa. The study has been performed at a hypothetic depth between 2000 and $2600 \mathrm{~m}$, consistent with the chosen thermodynamic conditions. Such a system is not intended to represent a real reservoir fluid, but it allows for studying a simple ternary asymmetric oil mixture composed of typical hydrocarbons at reservoir conditions.

Two cases have been studied:

- an isothermal case, in which only segregation occurs,

- a thermogravitation case in which the system is subject both to the gravity field and a geothermal gradient equals to $0.027 \mathrm{~K} . \mathrm{m}^{-1}$.

Using the scheme described previously, MD simulations have been performed for both cases. In addition, a conventional thermodynamic modeling using the Peng-Robinson EoS with volume shifts and cross interactions parameters has been carried out for the pure segregation case (Montel and Gouel 1985). At the reference conditions, EoS and MD simulations yield results on density in excellent agreement with each other, i.e. $\rho_{\mathrm{EoS}}=675.8 \mathrm{~kg} / \mathrm{m}^{3}$ and $\rho_{\mathrm{MD}}=676 \pm$ $0.1 \mathrm{~kg} / \mathrm{m}^{3}$.

As expected, when only the gravity field is active (segregation), $\mathrm{C}_{1}$ is enriched at the top of the fluid column (Fig. 2a) and $\mathrm{nC}_{12}$ is enriched at the bottom of the fluid column (Fig. 2c). Concerning $\mathrm{nC}_{4}$, one can notice a small enrichment (of about 0.001 in mole fraction) at the top of the fluid column (Fig. 2b). The concentration gradients obtained from the thermodynamic modeling are in good agreement with those coming from MD simulations despite 
a

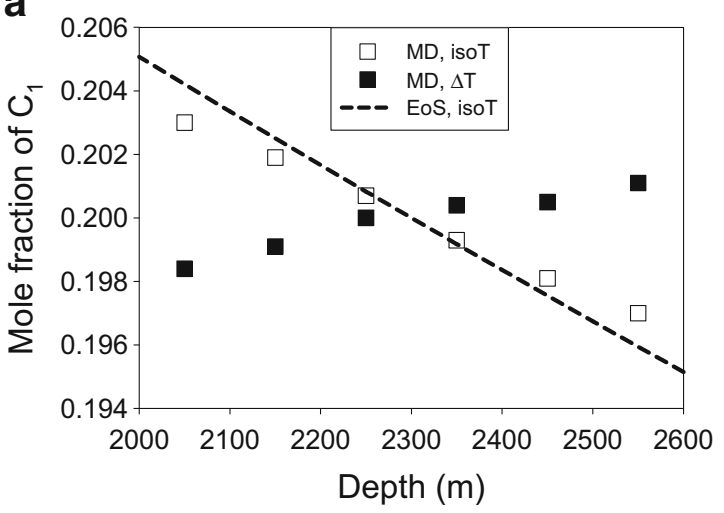

b

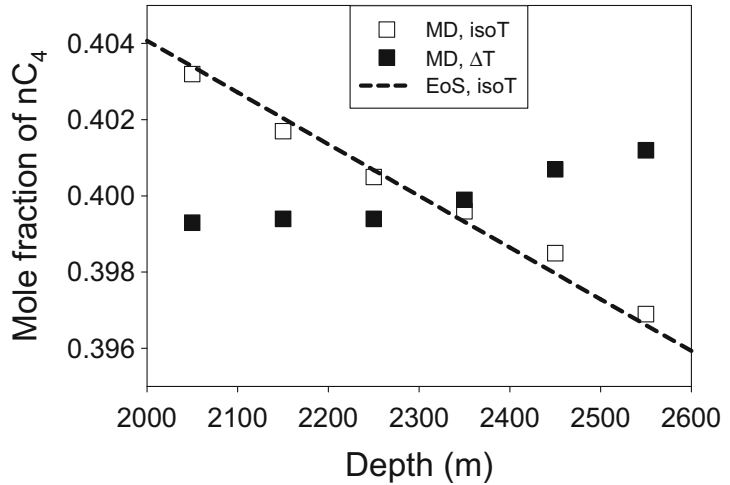

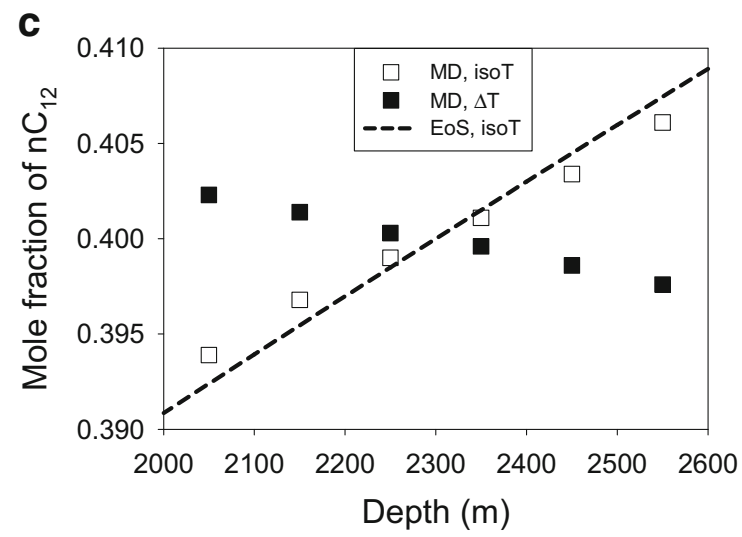

Fig. 2 Mole fractions versus depth in a ternary $C_{1}-n C_{4}-n C_{12}$ mixture subject to segregation (isoT) and thermogravitation ( $\left.\Delta \mathrm{T}\right)$ using $\mathrm{MD}$ simulations and classical thermodynamic modelling

quite different approaches. It is worth pointing out that the thermodynamic approach yields gradients that are slightly larger than those coming from the MD simulations, see Fig. 2.

When the geothermal gradient is added to segregation (thermogravitation) the MD simulation results are completely modified, see Fig. 2. Mole fraction gradients of $\mathrm{C}_{1}$, $\mathrm{nC}_{4}$ and $\mathrm{nC}_{12}$ are even reversed, compared to the purely gravitational segregation case, because of the thermodiffusion effect. More precisely, $\mathrm{nC}_{12}$ is slightly enriched at the top of the reservoir whereas the opposite is true for $\mathrm{C}_{1}$. This indicates that, at least for the $\mathrm{C}_{1}-\mathrm{nC}_{4}-\mathrm{nC}_{12}$ mixture, thermodiffusion has a larger impact than gravitational segregation on the vertical distribution of the components. Such a result confirms quantitatively the importance of thermodiffusion, in addition to gravitational segregation, when trying to estimate the initial vertical distribution of the components in a petroleum reservoir.

Another very interesting point concerns the density profile shown in Fig. 3. It appears that the density gradient is reversed when the thermal gradient is present as compared to the gravitational-only segregation case. This is due to the thermal expansion effect and to thermodiffusion leading to an enrichment of the heavier compounds
$\left(\mathrm{nC}_{12}\right)$ at the top of the fluid column, as seen in Fig. 2c. This reverse density gradient indicates that such a system would be unstable at the reservoir scale and would lead to convection. At the MD scale the system is, as already alluded to, always stable as the Rayleigh number remains always small and well below the critical threshold (Galliero and Montel 2008).

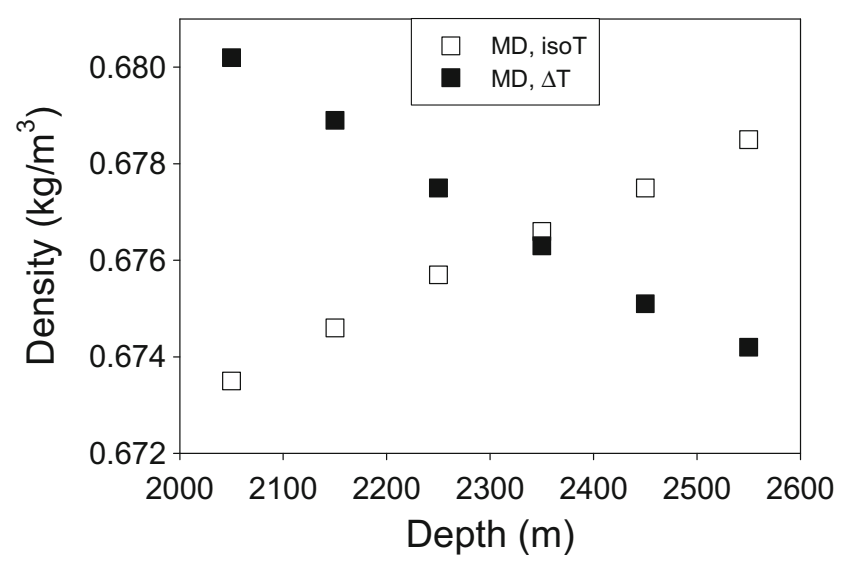

Fig. 3 Densities versus depth in a ternary $C 1-n C 4-n C 12$ mixture subject to segregation (isoT) and thermogravitation $(\Delta \mathrm{T})$ 

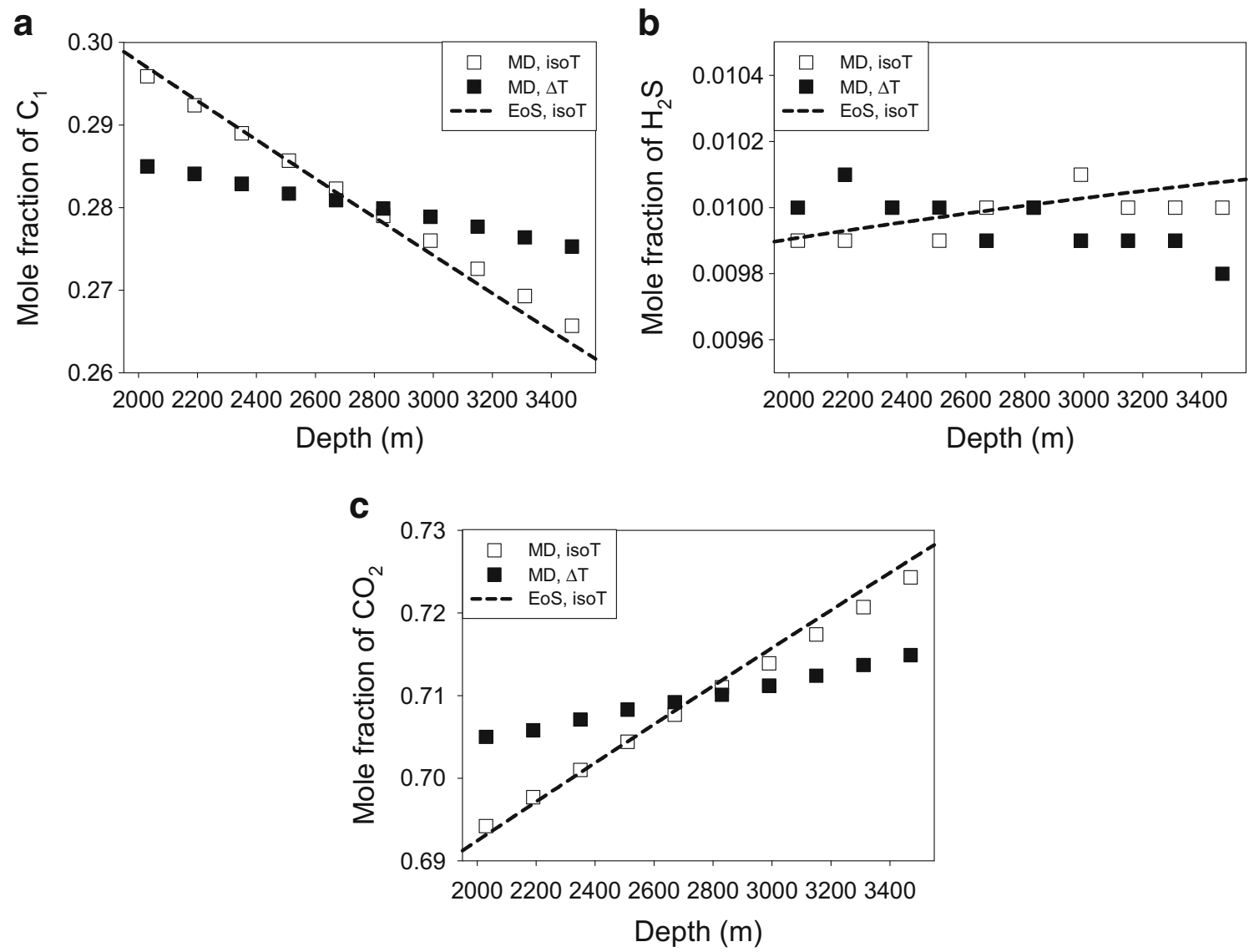

Fig. 4 Mole fractions versus depth in a ternary $\mathrm{CO}_{2}-\mathrm{C}_{1}-\mathrm{H}_{2} \mathrm{~S}$ mixture subject to segregation (isoT) and thermogravitation ( $\triangle \mathrm{T}$ ) using MD simulations and thermodynamic modelling

In this respect, we note that in real petroleum reservoirs, since hydrocarbon mixtures are within a porous matrix, permeability impacts the dynamic of the system, e.g. the Rayleigh-Darcy number should be considered instead of the Rayleigh number (Montel et al. 2007).

\section{Realistic Acid Gas Mixture}

As a second example, we have applied the approach to a real Far-East $\mathrm{CO}_{2}$ rich reservoir. The reservoir fluid is an acid gas mixture composed of $\mathrm{C}_{1}, \mathrm{CO}_{2}$ and $\mathrm{H}_{2} \mathrm{~S}$ with an average mole fraction equal to $0.28,0.71$ and 0.01 , respectively. Reference conditions at the mid column depth are a temperature of $443.15 \mathrm{~K}$ and a pressure of $40 \mathrm{MPa}$. The fluid column possesses an extremely high vertical extension equal to $1600 \mathrm{~m}$.

A methodology similar to that applied to the n-alkane mixture (see Section Synthetic Mixture Composed of Normal Alkanes.) has been used to deal with the segregation and the thermogravitation processes using a geothermal gradient equal to $0.03 \mathrm{~K} \cdot \mathrm{m}^{-1}$. At the reference conditions, EoS and MD simulations yield densities consistent with each other, i.e. $\rho_{\mathrm{EoS}}=408.9 \mathrm{~kg} / \mathrm{m}^{3}$ and $\rho_{\mathrm{MD}}=407.3 \pm 0.2$ $\mathrm{kg} / \mathrm{m}^{3}$.

Concerning the thermogravitation profiles, illustrated in Fig. 4, the thermal gradient induces a noticeable modification of the molar fraction profiles as a direct consequence of the thermodiffusion process. However, contrary to the $\mathrm{n}$-alkane mixture discussed previously, the molar fraction

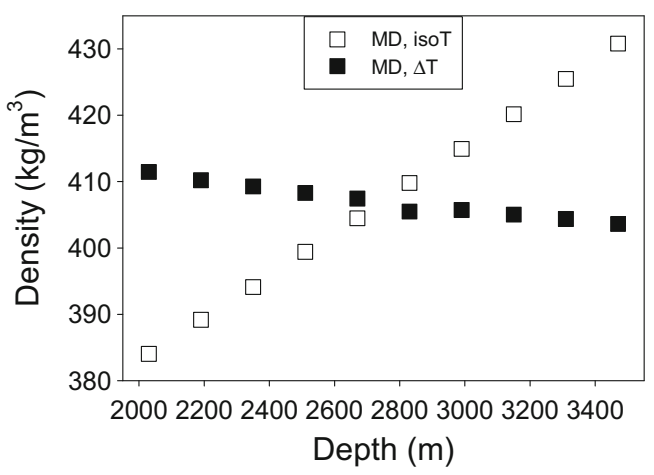

Fig. 5 Densities versus depth in an acid gas mixture subject to segregation (iso $\mathrm{T})$ and thermogravitation $(\Delta \mathrm{T})$ 
gradients are not reversed as compared to the purely gravitational segregation case. This implies that the gravity segregation is dominant over thermodiffusion for this acid gas mixture. This is expected as thermodiffusion is usually smaller in gases than in liquids (Wiegand 2004; Galliero et al. 2007b). Nevertheless, it is clear from these MD results that neglecting thermodiffusion could lead to large errors when estimating the initial state of the reservoir.

Similarly to what was found on the n-alkane mixture, the chosen thermal gradient, see Fig. 5, leads to an unstable fluid column (slightly reverse density gradient). However, after more detailed investigation of field data, the thermal gradient has been reevaluated and the current consensus is that $0.02 \mathrm{~K} . \mathrm{m}^{-1}$ is a more likely value. With such thermal gradient, the fluid column remains stable.

\section{Conclusions}

We have presented in this work a methodology to estimate the initial vertical distribution of the components in an idealized hydrocarbon reservoir subject to gravity alone (segregation) or combined with a geothermal gradient (thermogravitation). The presented approach made use of Molecular Dynamics simulations and can be used to test and complement the usual methods based on classical EoS. Furthermore, it can provide thermodiffusion coefficients and shed light on some of the underlying physical mechanism (stability) of the thermo-gravitational process.

This methodology has been applied to two mixtures, a synthetic oil composed of $\mathrm{C}_{1}, \mathrm{nC}_{4}$ and $\mathrm{nC}_{12}$ and a real acid gas composed of $\mathrm{CO}_{2}, \mathrm{C}_{1}$ and $\mathrm{H}_{2} \mathrm{~S}$. For the segregation case, an excellent agreement has been found between the proposed methodology and the results obtained by classical thermodynamic model (EoS). For thermogravitation case, MD simulations have shown that the thermodiffusion effect induced by the geothermal gradient is similar in magnitude (but opposite in sign) to that of gravitational segregation one. In addition, it has been observed that the density gradient can be reversed resulting in a higher density at the top of the fluid column than at the bottom, (i.e. an unstable situation) as a consequence of thermal expansion and thermodiffusion due to a presence of a geothermal gradient.

These numerical results confirm quantitatively the fact that thermodiffusion in multi-component mixtures noticeably impacts the initial vertical distribution of the species in a petroleum reservoir and should be taken into account to determine accurately the initial state of a reservoir. Although molecular dynamics and classical EoS can provide important information on such systems, it remains paramount to validate these modeling tools by actual measurements. The SCCO-SJ10 experiments will be a crucial step towards this goal.

Acknowledgments We gratefully acknowledge computational facilities provided by the University of Pau and the MCIA. This work has been supported by ESA through the SCCO project. The authors thank Total S.A. for permission to publish this paper. The Spanish teams (Mondragon Unibertsitatea and Universidad Complutense) are thankful to the TERDISOMEZ (FIS2014-58950-C2-1-P and FIS201458950-C2-2-P) of MINECO.

\section{References}

Allen, M.P., Tildesley, D.J.: Computer simulation of liquids. Clarendon Press, Oxford (1987)

Artola, P.A., Rousseau, B.: Thermal diffusion in simple liquid mixtures: What have we learnt from molecular dynamics simulations? Mol. Phys. 111, 3394-3403 (2013)

Assael, M.J., Goodwin, A.R.H., Vesovic, V., Wakeham, W.A.: Experimental Thermodynamics Volume IX: Advances in Transport Properties of Fluids. Royal Society of Chemistry, London (2014)

Berendsen, H.J.C., Postam, J.P.M., Van Gunsteren, W.F., DiNola, A., Haak, J.R.: Molecular Dynamics with coupling to an external bath. J. Chem. Phys 81, 3684-3690 (1984)

Bou-Ali, M.M., Ahadi, A., Alonso de Mezquia, D., Galand, Q., Gebhardt, M., Khlybov, O., Köhler, W., Larrañaga, M., Legros, J.C., Lyubimova, T., Mialdun, A., Ryzhkov, I., Saghir, M.Z., Shevtsova, V., Van Vaerenbergh, S.: Benchmark values for the Soret, thermodiffusion and molecular diffusion coefficients of the ternary mixture tetralin+isobutylbenzene+n-dodecane with $0.8-0.1-0.1$ mass fraction. Eur. Phys. J. E 38, 30 (2015)

Croccolo, F., Bataller, H., Scheffold, F.: A light scattering study of non equilibrium fluctuations in liquid mixtures to measure the Soret and mass diffusion coefficient. J. Chem. Phys 137, 234202 (2012)

de Groot, S.R., Mazur, P.: Nonequilibrium Thermodynamics. Dover, New York (1984)

Firoozabadi, A., Ghorayeb, K., Shukla, K.: Theoretical model of thermal diffusion factors in multicomponent mixtures. AICHE J. 46, $892-900$ (2000)

Galliero, G., Duguay, B., Caltagirone, J.P., Montel, F.: On thermal diffusion in binary and ternary mixtures by non-equilibrium molecular dynamics. Phil. Mag. 83, 2097-2108 (2003)

Galliero, G., Colombani, J., Bopp, P.A., Duguay, B., Caltagirone, J.P., Montel, F.: Thermal diffusion in micropores by molecular dynamics computer simulations. Physica A 361, 494-510 (2006)

Galliero, G., Nieto-Draghi, C., Boned, C., Avalos, J.B., Mackie, A.D., Baylaucq, A., Montel, F.: Molecular dynamics simulation of acid gas mixtures: A comparison between several approximations. Ind. Eng. Chem. Res 46, 5238-5244 (2007a)

Galliero, G., Bugel, M., Duguay, B., Montel, F.: Mass effect on thermodiffusion using molecular dynamics. J. Non-Equi. Thermodyn 32, 251-258 (2007b)

Galliero, G., Montel, F.: Nonisothermal gravitational segregation by molecular dynamics simulations. Phys. Rev. E 78, 041203 (2008)

Galliero, G., Srinivasan, S., Saghir, M.Z.: Estimation of thermodiffusion in ternary alkane mixtures using molecular dynamics and an irreversible thermodynamic theory. High Temp- High Press 38, 315-328 (2009) 
Galliero, G.: Equilibrium, interfacial and transport properties of nalkanes: Towards the simplest coarse grained molecular model. Chem. Eng. Res. Des 92, 2031-2037 (2014)

Georis, P., Montel, F., Van Vaerenbergh, S., Decoly, Y., Legros, J.C. In: Proceedings of the European Petroleum Conference, vol. 1, pp. 57-62 (1998)

Ghorayeb, K., Firoozabadi, A., Anraku, T.: Interpretation of the unusual fluid distribution in the Yufutsu gas-condensate field. SPE J. 8, 114-123 (2003)

Giraudet, C., Bataller, H., Croccolo, F.: High-pressure mass transport properties measured by dynamic near-field scattering of non-equilibrium fluctuations. Eur. Phys. J. E 37, 107 (2014)

Halldórsson, S., Stenby, E.H.: Isothermal gravitational segregation: Algorithms and specifications. Fluid Phase Equilib. 175(1-2), 175-183 (2000)

Hannaoui, R., Galliero, G., Hoang, H., Boned, C.: Influence of confinement on thermodiffusion. J. Chem. Phys 139, 114704 (2013)

Høier, L., Whitson, C.H.: Compositional grading-theory and practice. SPE Reserv. Eval. Eng. 4, 525-535 (2001)

Holt, T., Lindeberg, E., Ratkje, K.S.: The effect of gravity and temperature gradients on Methane distribution in Oil reservoirs. SPE Paper, 11761 (1983)

Hu, W.R., Zhao, J.F., Long, M., Zhang, X.W., Liu, Q.S., Hou, M.Y., Kang, Q., Wang, Y.R., Xu, S.H., Kong, W.J., Zhang, H., Wang, S.F., Sun, Y.Q., Hang, H.Y., Huang, Y.P., Cai, W.M., Zhao, Y., Dai, J.W., Zheng, H.Q., Duan, E.K., Wang, J.F.: Space Program SJ10 of Microgravity Research. Microgravity Sci. Tec. 26, 156169 (2014)

Kempers, L.J.T.M.: A comprehensive thermodynamic theory of the Soret effect in a multicomponent gas, liquid, or solid. J. Chem. Phys 115, 6330-6341 (2001)

Khlybov, O.A., Ryzhkov, I.I., Lyubimova, T.P.: Contribution to the benchmark for ternary mixtures: Measurement of diffusion and Soret coefficients in 1,2,3,4-tetrahydronaphthalene, isobutylbenzene, and dodecane onboard the ISS. Eur. Phys. J. E 38, 29 (2015)

Larrañaga, M., Bou-Ali, M.M., Alonso de Mezquía, D., Rees, D.A.S., Madariaga, J.A., Santamaría, C., Platten, J.K.: Contribution to the benchmark for ternary mixtures: Determination of Soret coefficients by the thermogravitational and the sliding symmetric tubes techniques. Eur. Phys. J. E 38, 28 (2015)
Leahy-Dios, A., Bou-Ali, M.M., Platten, J.K., Firoozabadi, A.: Measurements of molecular and thermal diffusion coefficients in ternary mixtures. J. Chem. Phys 122, 234502 (2005)

Montel, F., Gouel, P.L.: Prediction of compositional grading in a reservoir fluid column. SPE Paper, 14410 (1985)

Montel, F., Bickert, J., Lagisquet, A., Galliero, G.: Initial state of petroleum reservoirs: A comprehensive approach. J. Pet. Sci. Eng 58, 391-402 (2007)

Müller-Plathe, F.: A simple nonequilibrium molecular dynamics method for calculating the thermal conductivity. J. Chem. Phys 106, 6082-6085 (1997)

Ortiz de Zárate, J.M., Giraudet, C., Bataller, H., Croccolo, F.: Nonequilibrium fluctuations induced by the Soret effect in a ternary mixture. Eur. Phys. J. E 37, 77 (2014)

Perronace, A., Leppla, C., Leroy, F., Rousseau, B., Wiegand, S.: Soret and mass diffusion measurements and molecular dynamics simulations of n-pentane-n-decane mixtures. J. Chem. Phys 116, 3718$3729(2002)$

Platten, J.K., Costeseque, P.: The Soret coefficient in porous media. J. Porous Media 7, 317-329 (2004)

Sage, B.H., Lacey, W.N.: Gravitational concentration gradients in static column of hydrocarbon fluids. Trans. AIME 132, 120-131 (1939)

Shapiro, A., Stenby, E.H.: Factorization of transport coefficients in macroporous media. Transp. Porous Media 41, 305-323 (2000)

Touzet, M., Galliero, G., Lazzeri, V., Saghir, M.Z., Montel, F., Legros, J.C.: Thermodiffusion: from microgravity experiments to the initial state of petroleum reservoirs. Comptes Rendus - Mécanique 339, 318-323 (2011)

Urteaga, P., Bou-Ali, M.M., Alonso de Mezquía, D., Santamaría, J., Santamaría, C., Madariaga, J.A., Bataller, H.: Measurement of thermodiffusion coefficient of hydrocarbon binary mixtures under pressure with the thermogravitational technique. Rev. Sci. Instrum 83, 074903 (2012)

Van Vaerenbergh, S., Srinivasan, S., Saghir, M.Z.: Thermodiffusion in multicomponent hydrocarbon mixtures: Experimental investigations and computational analysis. J. Chem. Phys 131, 114505 (2009)

Whitson, C.H., Belery, P.: Compositional Gradients in Petroleum Reservoirs. SPE Paper, 28000 (1994)

Wiegand, S.: Thermal diffusion in liquid mixtures and polymer solutions. J. Phys. Condens. Matter 16, R357-R379 (2004) 